\title{
Theoretical approaches to the glass transition in simple liquids
}

\author{
CHANDAN DASGUPTA \\ Department of Physics, Centre for Condensed Matter Theory, Indian Institute of Science, \\ Bangalore 560 012, India \\ E-mail: cdgupta@physics.iisc.ernet.in
}

\begin{abstract}
Theoretical approaches to the development of an understanding of the behaviour of simple supercooled liquids near the structural glass transition are reviewed and our work on this problem, based on the density functional theory of freezing and replicated liquid state theory, are summarized in this context. A few directions for further work on this problem are suggested.
\end{abstract}

Keywords. Supercooled liquids; glass transition; density functional theory; liquid-state theories; replica method.

PACS Nos 64.70.Pf; 61.20.Lc; 61.20.Ne

\section{Introduction}

When a liquid is cooled to temperatures lower than its equilibrium crystallization temperature at a rate that is sufficiently fast to avoid crystallization, it enters a metastable supercooled state. The dynamical behaviour of supercooled liquids exhibits many interesting features, such as non-exponential, multi-stage decay of fluctuations and a rapid growth of relaxation times. As the temperature is decreased further, the viscosity of the supercooled liquid becomes so large that it behaves like a disordered solid for most purposes. This state of matter is called 'structural glass'. The glass transition temperature is conventionally defined as the temperature at which the viscosity reaches a value of $10^{13} \mathrm{P}$.

In spite of extensive experimental and theoretical investigations over several decades (see refs $[1,2]$ for reviews), there is still no consensus about a complete theoretical description of the experimentally observed behaviour of supercooled liquids near the structural glass transition. This article is concerned with theoretical studies of the behaviour of simple liquids with spherically symmetric interparticle interactions in the supercooled regime. After a brief description (in $\S 2$ ) of the most interesting aspects of the behaviour of such liquids near the structural glass transition, several recent theoretical approaches to understanding these features are discussed in $\S 3$. Our work on this problem, using the density functional theory of 
freezing and replicated liquid-state theory, is summarized and placed in the context of other theoretical developments in $\S \S 4$ and 5. Section 6 contains a few concluding remarks and suggestions for future work on this problem.

\section{Experimental features}

The most prominent feature of the dynamical behaviour of supercooled liquids is a rapid growth of the characteristic relaxation time as the temperature is decreased. This is reflected in a large number of experimentally measured quantities such as the viscosity and the dielectric relaxation time. The growth of the viscosity of the so-called fragile liquids [3] with decreasing temperature is usually described by the super-Arrhenius Vogel-Fulcher form [4]

$$
\eta(T) \propto \exp \left[B T_{0} /\left(T-T_{0}\right)\right]
$$

The value of the characteristic temperature $T_{0}$, obtained from fits to experimental data for $T>T_{\mathrm{g}}$, the conventionally defined glass transition temperature, is typically found to be about $10 \%$ lower than $T_{\mathrm{g}}$. Therefore, the attachment of physical significance to the temperature $T_{0}$ is dependent on the assumption that this form would continue to describe the growth of the viscosity all the way down to $T_{0}$ if one could somehow measure it for $T<T_{\mathrm{g}}$.

Another interesting feature of the behaviour of supercooled liquids is the observation [5] that the excess entropy defined as

$$
S_{\mathrm{ex}}(T) \equiv S_{l}(T)-S_{x t}(T)
$$

where $S_{l}(T)$ and $S_{x t}(T)$ are, respectively, the entropies of the supercooled liquid and the equilibrium crystal at temperature $T$, extrapolates to zero at a temperature $T_{\mathrm{K}}$ (called the Kauzmann temperature) which is close to the temperature $T_{0}$ at which $\eta$ is supposed to diverge, according to eq. (1). This observation suggests a close relation between the growth and possible divergence of $\eta$ and the vanishing of $S_{\text {ex }}$. Such a relation between these two quantities was postulated many years ago by Adam and Gibbs [6]:

$$
\eta(T) \propto \exp \left[C /\left(T S_{\mathrm{ex}}(T)\right)\right]
$$

where $C$ is a constant. This relation suggests that the experimentally observed behaviour for $T>T_{\mathrm{g}}$ may be the precursor of a thermodynamic 'entropy-vanishing' transition that would take place at $T=T_{0}=T_{\mathrm{K}}$ if thermodynamic equilibrium (in a restricted sense, excluding the regions of phase space near crystalline states) could be maintained down to this temperature.

A theoretical description of the dynamics of supercooled liquids, known as the mode-coupling theory [7] provides a fairly accurate account of the results of experiments [8] in the temperature range that covers the first few decades of the growth of the viscosity. The simplest version of mode-coupling theories predicts a power-law divergence of the viscosity at an ideal glass transition temperature $T_{c}$. However, the value of $T_{c}$ obtained from fits of higher-temperature data to the mode-coupling 
form turns out to be substantially higher than $T_{\mathrm{g}}$. Thus, conventional mode coupling theories do not provide a correct description of the behaviour at temperatures close to or lower than $T_{c}$. The dynamical behaviour exhibits a crossover near $T_{c}$, and this crossover plays an important role in a number of theoretical approaches, as discussed below.

\section{Review of theoretical approaches}

Some of the recent developments in the theory of glass transitions are briefly described in this section. In each case, new insights that have been gained are discussed and questions that require further clarification are pointed out.

\section{1 'Spin-glass-like' theories}

This theoretical approach is based on analogies between the phenomenology described above and theoretical results for a class of mean-field spin glass models. These analogies were first pointed out by Kirkpatrick, Thirumalai and Wolynes [9-12] several years ago. More recently, there have been many new developments [13-16] in theoretical descriptions based on this analogy. The spin glass models relevant in this context are Potts glasses and Ising spin glasses with multispin interactions. Analytic studies of these mean-field models with infinite-range interactions bring out a remarkable correspondence between their behaviour and that of supercooled liquids near the glass transition. These spin glass models exhibit a dynamical transition to non-ergodic behaviour at $T=T_{d}$. The dynamics as $T$ approaches $T_{d}$ from above is described by the ideal version of mode coupling theory. The thermodynamic behaviour for $T<T_{d}$ is governed by an exponentially large number of local minima of the free energy. The canonical free energy in this regime is given by

$$
F(T)=-T \ln \sum_{k} \exp \left(-F_{k} / T\right)=\left\langle F_{k}\right\rangle-T S_{c}(T),
$$

where $F_{k}$ is the free energy of the $k$ th minimum, and $S_{c}(T)$ is the extensive configurational entropy or complexity of the free-energy minima. The configurational entropy per spin goes to zero at a temperature $T_{s}<T_{d}$. A thermodynamic transition described by one-step replica symmetry breaking takes place at this temperature. A connection with the glass transition is made by relating the dynamical transition at $T_{d}$ to the ideal glass transition of mode coupling theories (it is argued that the dynamical transition in the mean-field models becomes a crossover in real systems with short-range interactions), and the thermodynamic transition at $T_{s}$ to the putative entropy-vanishing transition at $T_{0}=T_{\mathrm{K}}$.

This analogy has been successfully used to obtain qualitative descriptions of a variety of interesting features of the behaviour of supercooled liquids. For example, there has been a lot of interest in recent years in the study of dynamical heterogeneity in supercooled liquids [17-19], which describes the spatial inhomogeneity of the local relaxational dynamics. A four-point correlation function, introduced by us $[20,21]$ 
several years ago, has received a lot of attention in this context. This four-point correlation function is defined as

$$
\begin{aligned}
g_{4}(\mathbf{r}, t)= & \langle\delta \rho(0,0) \delta \rho(0, t) \delta \rho(\mathbf{r}, 0) \delta \rho(\mathbf{r}, t)\rangle, \\
& -\langle\delta \rho(0,0) \delta \rho(0, t)\rangle\langle\delta \rho(\mathbf{r}, 0) \delta \rho(\mathbf{r}, t)\rangle,
\end{aligned}
$$

where $\delta \rho(\mathbf{r}, t)$ represents the deviation of the local density at point $\mathbf{r}$ and at time $t$ from its average value. This function measures the correlation between density relaxations at two points separated by $\mathbf{r}$. Numerical studies $[19,22]$ show that the time-dependent susceptibility corresponding to this correlation function peaks at a characteristic value of $t$ and both the value of $t$ at which this peak occurs and the value of the susceptibility at the peak increases as the temperature is decreased. The results of the calculation [22] of a similar quantity for one of the mean-field spin glass models show remarkable similarity with the results of simulations, indicating that this description captures, at least qualitatively, the essential characteristics of the spatially heterogeneous dynamics of supercooled liquids.

In spite of such successes, several questions need to be addressed before one can conclude that these theories provide a correct description of the behaviour near the glass transition in simple liquids. Some of these questions are:

1. The spin glass models have quenched disorder which is not present in supercooled liquids. This is not a serious problem, since several studies [23,24] have shown that the presence of frustration in models without quenched disorder can lead to effects very similar to those found in systems with quenched disorder.

2. Calculations for simple models of liquids are necessary to validate the analogy with the behaviour of the spin glass models. Some of the calculations based on density functional theory and liquid-state theories, to be discussed in $\S \S 4$ and 5 , address this issue.

3. The spin glass models for which the analytic calculations mentioned above have been carried out are all mean-field ones with infinite-range interactions. It is not clear whether the results obtained from these calculations remain valid for physical systems with short-range interactions. This question acquires particular significance from the observation [25] that, unlike the infinite-range model, the 10-state short-range Potts glass does not exhibit any finite-temperature dynamical or static phase transition in three dimensions. This is a serious problem which is discussed further in $\S 6$.

4. Calculations for the mean-field spin glass models do not provide any information about the dynamics arising from transitions among the large number of free-energy minima for $T<T_{d}$. If one identifies $T_{d}$ with the ideal glass transition temperature $T_{c}$ of mode coupling theories, then the dynamics for $T<T_{c}$ in real systems, for which the free-energy barriers do not diverge in the thermodynamic limit, is expected to be governed by such transitions. It has been suggested $[12,26]$ that an entropically driven nucleation mechanism may lead to a rapid growth of relaxation times in this temperature regime, leading to a Vogel-Fulcher divergence of the viscosity at the temperature where the configurational entropy of the collection of free-energy minima becomes zero. 
The arguments that lead to this conclusion are suggestive, but not completely conclusive [27]. This aspect requires further clarification.

\subsection{The 'inherent structures' approach}

This primarily numerical approach is based on the premise that the equilibrium and dynamical properties of a classical supercooled liquid at sufficiently low temperatures ( $T$ close to or lower than $T_{c}$ ) can be understood in terms of the properties of the 'inherent structures' (local minima of the potential energy) sampled by the system as it evolves under specified external conditions. This approach, originally proposed by Goldstein [28] and developed by Stillinger and Weber [29,30], has been extended in recent years by several researchers [31-40] to studies of a variety of properties of simple model liquids in the supercooled regime. In this approach, the potential energy landscape of a system of particles interacting via a specified model potential is described in terms of the inherent structures and their basins of attraction. The basin of attraction of an inherent structure is defined as the collection of all states that converge to it under a specified deterministic energy minimization procedure such as the conjugate gradient method. The basins of attraction of the inherent structures provide a partitioning of the configuration space of the liquid. The partition function of a system of $N$ particles with density $\rho$ at temperature $T$ may then be written as

$$
Q(N, \rho, T)=\int \mathrm{d} \Phi \exp \left[-\left(\Phi+F_{\text {bas }}(\Phi, T)\right) / T+S_{c}(\Phi)\right],
$$

where $\Phi$ is the potential energy, $F_{\text {bas }}(\Phi, T)$ is the free energy associated with the states in the basin of attraction of an inherent structure of energy $\Phi$ (it is assumed that this quantity is the same for all such inherent structures), and $S_{c}(\Phi)$, the configurational entropy of inherent structures with energy $\Phi$ given by

$$
S_{c}(\Phi) \equiv \ln \Omega(\Phi),
$$

where $\Omega(\Phi)$ is the 'density of states' of the inherent structures. At temperatures close to or lower than $T_{c}$, the average energy of the inherent structures sampled by the system decreases as the temperature is decreased [31]. The configurational entropy at temperature $T$ is given by

$$
S_{c}(T)=\int \mathrm{d} \Phi S_{c}(\Phi) P(\Phi, T),
$$

where $P(\Phi, T)$ is the probability density that the system samples inherent structures with energy $\Phi$ during its time evolution at temperature $T$.

Both $S_{c}(\Phi)$ and $S_{c}(T)$ can be determined numerically by appropriate sampling methods. Several studies $[33,34]$ have shown that the numerically determined $S_{c}(T)$ extrapolates to zero at temperature $T_{\mathrm{K}}$ that is close to the temperature $T_{0}$ extracted from Vogel-Fulcher fits to dynamical data for the same system (see, however, ref. [36] that reaches a different conclusion about the behaviour of $S_{c}(T)$ ). The Adam-Gibbs relation of eq. (3) is found [37] to be well-satisfied by the numerical data. These results provide support to the validity of the 'spin-glass-like' 
theories discussed above: the free-energy minima postulated in these theories are not very different from the inherent structures at sufficiently low temperatures. The inherent structures approach has also been used to study various other features of the behaviour of supercooled liquids. These include the crossover in the dynamical behaviour near the mode-coupling temperature $T_{c}[38,39]$, the 'fragility' parameter [3] that characterizes the growth of the viscosity with decreasing temperature [40], and the aging behaviour after a quench from high temperatures to the supercooled state [41]. While a lot of interesting results have been obtained from these studies, the interpretation of some of these results is still controversial.

It is clear from the discussion above that the inherent structures approach has been quite useful in understanding the behaviour of simple model liquids near the glass transition. However, for further progress, it is necessary to clarify a few questions about this approach. These include:

1. Numerical studies based on this approach are all carried out at temperatures higher than or close to $T_{c}$ because it is very difficult to equilibrate the system at lower temperatures. Since $T_{c}$ is substantially higher than the putative phase transition temperature $\left(T_{\mathrm{K}} \simeq T_{0}\right)$, any conclusion drawn from these studies about the occurrence of such a transition has to be based on extrapolations whose validity can be questioned.

2. Several years ago, Stillinger [42] presented rather convincing arguments which showed that the configurational entropy defined in eq. (8) cannot vanish at any non-zero temperature in substances with limited molecular weight and conventional intermolecular interactions. Therefore, a different definition of the configurational entropy is required for the existence of a non-zero Kauzmann temperature $T_{\mathrm{K}}$. It should be noted in this context that the identification of the experimentally measured excess entropy defined in eq. (2) with the configurational entropy defined in eq. (7) (or with the complexity defined in eq. (4) in spin-glass-like theories) is an assumption for which complete justification is not yet available.

3. The relation between the slow dynamics of supercooled liquids and the topography of its potential energy landscape is not very clear. While it is intuitively appealing to attribute the slow dynamics to thermally activated transitions between neighboring inherent structures, this simple picture does not provide a quantitative account of the dynamics observed in simulations. It has been suggested by Doliwa and Heuer [43-45] that the time scale of structural relaxation is related to the heights of energy barriers between different 'metabasins' which are defined as groups of strongly correlated inherent structures. The concept of metabasins may also be relevant in obtaining an alternative definition of the configurational entropy that would not be subject to Stillinger's argument against the existence of a non-zero Kauzmann temperature. However, the definition of metabasins proposed by Doliwa and Heuer is not completely satisfactory because it requires monitoring the actual time evolution of the system. Further work in identifying the metabasins (or similar structures in the energy landscape) from features in the topography of the energy landscape would be very useful. 


\subsection{Other scenarios}

Two other approaches that have received some attention in recent years are briefly described in this section. The first one is based on studies of spin or lattice-gas models with constrained kinetics [46,47]. The equilibrium behaviour of these models is trivial (in particular, there is no possibility of a thermodynamic phase transition), but their dynamics shows many interesting features that are qualitatively similar to the behaviour found in supercooled liquid. On the basis of these observations, it has been argued $[48,49]$ that the behaviour of supercooled liquids near the (experimentally defined) glass transition is the manifestation of a dynamical 'blocking' process that does not involve any underlying thermodynamic transition. While early numerical work on the kinetically constrained models suggested the occurrence of a dynamical transition with diverging relaxation time at a non-zero temperature, recent theoretical work [50] has ruled out this possibility. Nevertheless, the similarity of the dynamical behaviour of supercooled liquids with simulation results for these models suggests that the possibility that the glass transition is purely dynamical in origin should be taken seriously. However, there are serious questions [51] about whether this description can reproduce some of the experimentally observed thermodynamic behaviour near the glass transition. Also, this description would not be able to explain interesting connections between thermodynamic and dynamical quantities, such as the Adam-Gibbs relation of eq. (3).

The second approach [52-55] invokes the concept of 'avoided critical behaviour' to explain the phenomenology of the glass transition. This description is based on an analogy with the behaviour of a class of spin models with short-range interactions that tend to induce long-range order and weak, long-range interactions that induce frustration and prevent the occurrence of order. At temperatures lower than $T^{*}$ at which ordering would have taken place if the frustrating interactions were absent, the system breaks up into 'frustration limited domains' and the relaxation time in this regime exhibits super-Arrhenius growth according to the following form:

$$
\tau(T)=\tau_{0} \exp [E(T) / T], \quad E(T)=E_{0}+C\left(1-T / T^{*}\right)^{\alpha},
$$

where $\tau_{0}, E_{0}$ and $C$ are constants, and $\alpha=8 / 3$. This form provides [52] a reasonable description of the experimental data for several commonly studied liquids. However, in order to validate this description, it would be necessary to elucidate the nature of the short-range ordering and long-range frustrating interactions in liquids by carrying out explicit calculations for models of interacting particles.

\section{Glassy local minima of free energy functionals}

As discussed above, an intuitively appealing description of the behaviour of simple liquids in the supercooled regime is based on the premise that an exponentially large number of local minima of the free energy are present at sufficiently low temperatures (or at sufficiently high densities in systems, such as hard-sphere fluids, for which the density is the control parameter). The density functional theory of non-uniform liquids, in which the free energy is expressed as a function of the time-averaged local density, provides a convenient starting point for investigating 
whether this actually happens in simple model systems. Studies based on the inherent structures approach described above have shown that simple model liquids do exhibit a large number of local minima of the potential energy. While these potential energy minima are analogous to free energy minima at very low temperatures, there are important differences between the potential energy landscape and the free energy landscape. Properties of free energy minima are expected to change as the temperature is changed, and nontrivial minima of the free energy are expected to be present only at sufficiently low temperatures. In contrast, the inherent structures are, of course, defined without any reference to the temperature - the dependence of physical quantities on the temperature arises in this description from the fact that different groups of inherent structures are sampled at different temperatures. Thus, the properties of the free energy landscape of model liquids are not simply related to those of their potential energy landscape.

Density functional theories have been used extensively in the past to study the crystallization of simple liquids. In this description, the high-temperature liquid state of the system is represented by a minimum of the free energy functional with uniform density $\left(\rho(\mathbf{r})=\rho_{0}\right.$, the average density). As the temperature is decreased (or the average density is increased), the free energy functional develops a new minimum representing the crystalline state, characterized by a periodic spatial modulation of the local density. The question of interest in the context of the glass transition is whether a large number of 'glassy' (inhomogeneous but not periodic) local minima of the free energy also appear as the temperature is decreased below the equilibrium crystallization temperature.

This question has been addressed in several studies [56-59] in which certain assumptions were made about the structure of the glassy local minima of the free energy. While the results obtained in these studies are interesting and suggestive, it is not clear whether the glassy minima found in these studies would survive as local minima of the free energy if the constraints implied by the assumptions about their structure are removed. We have used unconstrained numerical minimizations of the Ramakrishnan-Yussouff (RY) [60] free-energy functional to study various properties of the free energy landscape of the hard-sphere system. The RY free energy functional has the form

$$
\begin{aligned}
\beta \Delta F= & \int \mathrm{d} \mathbf{r}\left[\rho(\mathbf{r}) \ln \left(\rho(\mathbf{r}) / \rho_{0}\right)-\rho(\mathbf{r})+\rho_{0}\right] \\
& -\frac{1}{2} \int \mathrm{d} \mathbf{r} \int \mathrm{d} \mathbf{r}^{\prime} c\left(\left|\mathbf{r}-\mathbf{r}^{\prime}\right|\right)\left[\rho(\mathbf{r})-\rho_{0}\right]\left[\rho\left(\mathbf{r}^{\prime}\right)-\rho_{0}\right],
\end{aligned}
$$

where $\Delta F$ is the difference between the (grand-canonical) free energy of an inhomogeneous state with time-averaged local density $\rho(\mathbf{r})$ and that of the homogeneous liquid with density $\rho_{0}, \beta$ is the inverse temperature, and $c(r)$ is the direct pair correlation function [61] of the uniform liquid with density $\rho_{0}$. Our approach [62] involves spatial discretization and direct, unconstrained, numerical minimization of the free energy written as a function of a large number of local density variables. The Percus-Yevick approximation [61] for the direct correlation function of the hard-sphere fluid is used in all our calculations. The control parameter for the hard-sphere system is the dimensionless density $n^{*} \equiv \rho_{0} \sigma^{3}(\sigma$ is the hard sphere diameter), which is related to the packing fraction $\eta_{f}$ by $\eta_{f}=\pi n^{*} / 6$. The 
Percus-Yevick approximation is known to provide an accurate description of the structure of the hard-sphere fluid if the value of $n^{*}$ is not very large.

We find, in agreement with earlier studies [56,57], that the RY free energy functional exhibits a large number of glassy local minima for densities substantially higher than the equilibrium crystallization density. The free energies of these minima are lower than that of the uniform liquid if the value of $n^{*}$ is sufficiently high, but are higher than that of the fcc crystalline state at all densities. We have carried out several studies [62-71] in which various properties of this glassy free energy landscape are investigated and the role of these glassy minima in the dynamical behaviour of the system is analysed. The main results obtained from these studies are summarized below.

1. Structural properties of the glassy minima: From investigations of spatial correlations of the local density and the distribution of the 'bond angles' that characterize the local packing geometry, we find $[62,65]$ that the glassy minima have amorphous structure similar to that found in simulations of the hard sphere system.

2. Role of the glassy minima in the dynamics of the system: From numerical studies [63-66] of the equations of fluctuating hydrodynamics in which the RY free energy functional is incorporated, we find that the dynamics is described by small fluctuations near the uniform liquid minimum if the density is sufficiently low. At higher densities, the dynamics is found to be governed by transitions between different glassy minima. The time scale of transitions between different glassy minima increases $[67,68]$ with the density.

3. Topography of the free energy landscape: The configurational entropy of the free energy minima is found [70,71] to decrease as the density is increased. Since we cannot enumerate all the glassy local minima, our study does not provide information about how the configurational entropy depends on the number of particles, or about whether it goes to zero at the 'Kauzmann' density. The distribution of the overlap between different glassy minima exhibits features similar to those expected in spin-glass-like theories.

4. Height of free energy barriers between different local minima: Using a 'microcanonical' Monte Carlo method, we have estimated [69-71] the heights of free energy barriers that separate different glassy minima of the free energy. Typical results are shown in figure 1. We find that the barrier height increases with increasing density, and that this growth is consistent with a Vogel-Fulcher form appropriate for the hard sphere system [72] for which the density (not the temperature) is the control parameter.

These results provide qualitative explanations of several features observed in molecular dynamics simulations [72,73] of dense hard sphere fluids. Most of our results are similar to those expected in spin-glass-like theories discussed in $§ 3.1$. Thus, our studies provide support to the validity of this description. Our earlier studies of the free energy landscape were carried out using relatively small sample sizes $(\sim 100$ particles $)$ and somewhat coarse discretization scales $(\sim 0.2 \sigma)$. This made it difficult to compare our results quantitatively with those of simulations. Recently, increased computational capabilities have allowed us to carry out similar 
calculations [74] for larger systems and smaller discretization scales. Figure 2 shows a sample of our recent results: the free energy of the fcc crystal and that of a glassy local minimum, obtained for a system containing $\sim 500$ particles, are plotted in this figure as functions of the dimensionless density $n^{*}$. The discretization scale used in this calculation is close to $\sigma / 20$. For these parameter values, our results for

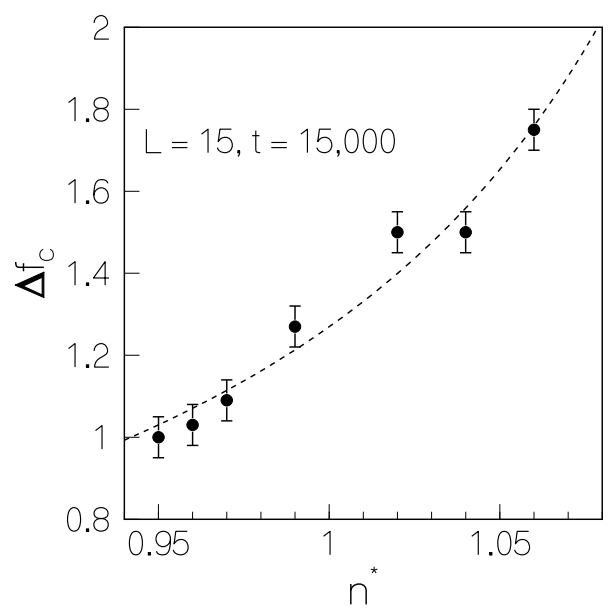

Figure 1. Height of the effective free energy barrier per particle (in units of $T$ ) as a function of the dimensionless density $n^{*}$. The dashed line shows a fit to the Vogel-Fulcher form for hard sphere fluids, $\Delta f_{c} \propto 1 /\left(n_{0}^{*}-n^{*}\right)$, with $n_{0}^{*} \simeq 1.2$.

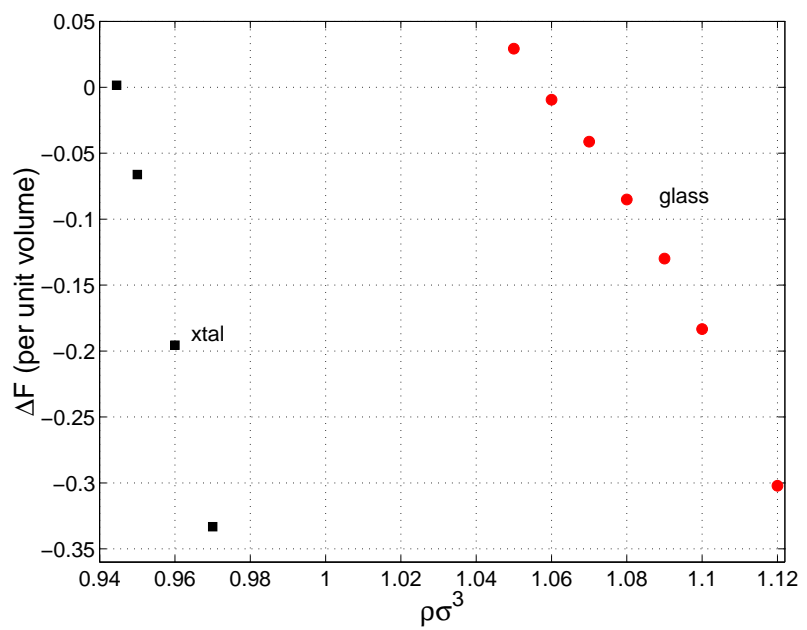

Figure 2. Free energies of the fcc crystal (squares) and of a glassy local minimum (circles), measured from that of the uniform liquid, as functions of the dimensionless density $n^{*}=\rho \sigma^{3}$. 
the crystallization transition are very close to those obtained from simulations. As shown in figure 2, the free energy of the glassy minimum crosses zero (i.e. becomes lower than that of the uniform liquid) near $n^{*}=1.06$, which is close to the value of the density at which the hard-sphere fluid begins to exhibit [59] interesting dynamics. We have also compared the density distribution at the glassy minimum with that obtained from molecular dynamics simulations and found in agreement with recent suggestions [59], that the $\rho(\mathbf{r})$ at a glassy minimum represents short-time averages of the local density. These observations confirm the relevance of the glassy minima in describing the dynamical behaviour in the supercooled regime.

\section{Replicated liquid-state theory}

The description of the properties of supercooled liquids in terms of the glassy local minima of a model free energy functional is analogous to inhomogeneous CurieWeiss molecular field theory for disordered spin systems such as spin glasses. A more sophisticated form of molecular field theory, in which the 'Onsager reaction term' is included, leads to the exact Thouless-Anderson-Palmer equations [75] for infinite-range spin glass models such as the ones mentioned in $\S 3.1$. An alternative approach to studies of such spin glass models is provided by the replica formalism [75]. It is, therefore, interesting to enquire whether the replica formalism can be used to study the structural glass transition. Our work along this line is summarized below.

Several years ago, we showed [76] that the standard methods of liquid state theory [61] can be combined with the replica formalism to study the equilibrium behaviour of liquids in the presence of an external random potential. While the formalism developed in this work was for a system of superconducting vortices in the presence of a random pinning potential, it can be readily generalized to other systems. Consider a classical liquid in a quenched Gaussian random potential $\Phi(\mathbf{r})$ with zero average and correlations given by

$$
\left[\Phi(\mathbf{r}) \Phi\left(\mathbf{r}^{\prime}\right)\right]_{\mathrm{av}}=V\left(\mathbf{r}-\mathbf{r}^{\prime}\right),
$$

where $[\cdots]_{\text {av }}$ represents an average over the probability distribution of the random potential. In the replica formalism for dealing with the quenched disorder, $n$ replicas of the system, labeled by $a=1, \ldots, n$ are introduced, with the requirement that the $n \rightarrow 0$ limit has to be taken to obtain physical quantities averaged over different realizations of the disorder. In the liquid-state problem, this is equivalent to having a mixture of $n$ 'kinds' of particles, each kind labeled by the replica index a. Averaging over the random potential leads to a new interaction of the form $-\beta V\left(\mathbf{r}_{i}^{a}-\mathbf{r}_{j}^{b}\right)$. This interaction is attractive. Thus, the problem reduces to one involving an $n$-component mixture with attractive interaction between particles of different components. Standard methods of liquid-state theory, e.g. the hypernetted chain (HNC) approximation [61] can be generalized [76] to treat such $n$-component mixtures, and the $n \rightarrow 0$ limit can be taken analytically. Operationally, this corresponds to the extremization of a free energy $F\left[\left\{g^{a b}(r)\right\}\right]$ written as a functional of 
the two-point density correlation functions $\left\{g^{a b}(r)\right\}$ of the $n$-component mixture. If replica symmetry is assumed, then one has to consider two such functions:

$$
\begin{aligned}
g^{a b}(r) & =g_{1}(r) \quad \text { if } a=b \\
& =g_{2}(r) \quad \text { if } a \neq b
\end{aligned}
$$

Physically, the function $g_{1}(r)$ represents the disorder-averaged, equal-time, twopoint correlation function of the local density, and $g_{2}(r)$ represents the two-point correlation of the disorder-induced spatial variation of the time-averaged local density. A recent study [77] has shown that the results for these two functions obtained from replicated liquid state theory provide a very good account of the behaviour observed in simulations of simple liquids in a random potential.

Since there is no external random potential in the structural glass problem, it is necessary to figure out how this formalism can be used to study the structural glass transition. This was done by Mézard and Parisi [78] who showed that the equations of replicated liquid state theory (specifically, the equations obtained in the HNC approximation) admit one-step replica symmetry breaking, similar to that found in the mean-field spin glass models discussed in $\S 3.1$, even if no external random potential is present. This one-step replica symmetry breaking transition is interpreted as a thermodynamic glass transition.

We have used [79] this formalism to map out the phase diagram of a hard sphere fluid in the presence of an external random potential. Our results for the phase diagram in the density-disorder plane are shown in figure 3 . In the absence of a

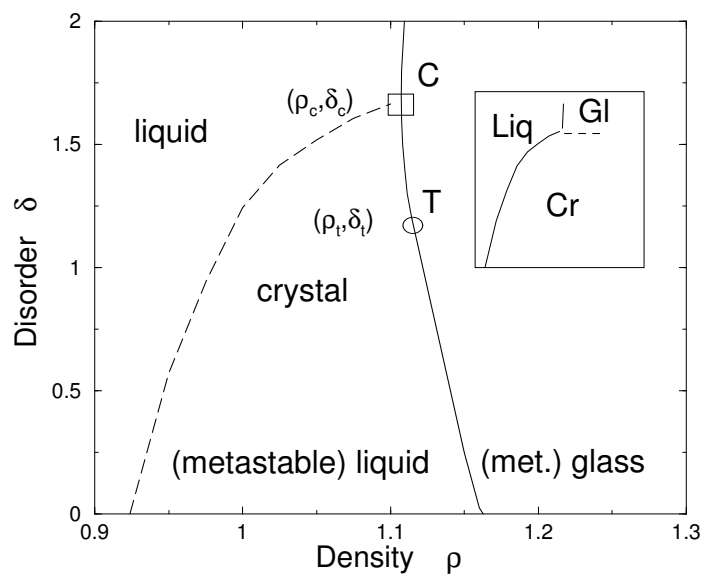

Figure 3. Phase diagram of a hard sphere fluid in a random potential, obtained from replicated liquid state theory and replicated density functional theory. Three phases, weakly disordered crystal, inhomogeneous liquid, and strongly disordered glass, are shown in the dimensionless density $\left(\rho=n^{*}\right)$ vs. disorder strength $(\delta)$ plane. The first-order crystallization line (dashed line) meets the glass transition line at the multicritical point $\mathrm{C}$. The nature of the glass transition changes from random first-order to continuous at the tricritical point $\mathrm{C}$. The inset shows only the equilibrium phases. 
random potential $(\delta=0)$, the calculations show, in agreement with the results of ref. [78], a thermodynamic glass transition, signalled by the occurrence of one-step replica symmetry breaking, near dimensionless density $n^{*}=1.16$. The calculations also show the occurrence of a dynamical transition at a slightly lower value of $n^{*}$. This is very similar to the behaviour found in the mean-field spin glass models discussed in $\S 3.1$.

In our study of the phase diagram in the density-disorder plane, we used a replicated version of the RY density functional to map out the line across which a transition from the liquid to a weakly disordered crystal occurs. As shown in figure 3, this transition is replaced by a continuous liquid-to-glass transition if the disorder strength exceeds a critical value. Thus, this calculation predicts a thermodynamically stable glass phase in liquids with sufficiently strong external disorder. We have also used [80] direct numerical minimization of the RY free energy functional for the same system to map out its phase diagram. The results of this study are qualitatively very similar to those obtained in our replica-based analysis. Thus, these results suggest that the replicated liquid state theory and the density functional theory provide very similar descriptions of the behaviour of dense supercooled liquids.

\section{Concluding remarks}

The results described in the last two sections suggest that the RY density functional theory and replicated liquid-state theories provide a mutually consistent description similar to the 'spin-glass-like' theories of the glass transition. However, one must remember that all these descriptions are mean-field in nature. Both density functional theory and replicated liquid state theory consider the minima of approximate free energy functionals (a functional of the local density in the first case, and a functional of the density correlators in the second case) - fluctuations about the minima are not taken into account in these descriptions. The same is, of course, true for the 'spin-glass-like' theories based on studies of infinite-range models. Other studies of the glass transition [81-85] based on liquid-state theories also appear to be [86] mean-field-like in nature. Thus, although an intuitively appealing description of the glass transition emerges from these theories, it is necessary to understand the effects of fluctuations, expected to be very important in three-dimensional systems with short-range interactions, before one can conclude that these theories are correct.

Traditionally, numerical studies using finite-size scaling have played a very important role in determining whether the behaviour of physical systems with short range interactions is analogous to that predicted by mean-field theory. Similar studies of the structural glass transition would obviously be very useful. We have carried out a few exploratory studies $[21,87]$ in this direction. In one of these studies [21], we found that the system-size dependence of the dynamics of a simple model liquid exhibits an unexpected feature: the relaxation time at temperatures close to the mode-coupling temperature $T_{c}$ increases as the system size is decreased. The same behaviour, but on a more dramatic scale, has been observed in the simulations of ref. [88]. This is very different from the usual finite-size scaling behaviour seen in 
simulations [89] of a mean-field Potts glass model near its dynamical transition. This difference between the observed behaviours of simple liquids and mean-field models needs further study and explanation. It is interesting to note in this context that another numerical study [90] of a different model liquid finds similar behaviour (but to a lesser degree) at relatively high temperatures, but a different trend (relaxation time increasing with system size) at lower temperatures. It would be very useful to extend such studies to lower temperatures (closer to the putative glass transition temperature). Development of algorithms that would allow equilibration of the system at lower temperatures is clearly very important in this context. A 'domain-wall scaling' analysis of the glass transition in a model liquid, carried out by us [87] several years ago, did not find any evidence for a finite-temperature thermodynamic transition. Similar studies for larger systems, using more efficient algorithms for equilibrating the system and locating potential-energy minima with the lowest energies, would help in understanding the true nature of the glass transition.

On the analytic side, methods for taking into account the effects of fluctuations in density functional and liquid state theories need to be developed. Other areas in which analytic progress would be welcome include incorporation of the effects of a rugged free energy landscape in the mode coupling description of the dynamics, and explorations of the alternative scenarios mentioned in $\S 3.3$.

\section{Acknowledgements}

This work was carried out in collaboration with O T Valls, S Ramaswamy, A V Indrani, M K Phani, G I Menon, S S Ghosh, L M Lust, F Thalmann, D Feinberg, $\mathrm{P}$ Chaudhuri and S Karmakar. The author is grateful to S Sastry for many interesting discussions.

\section{References}

[1] C A Angell, Science 267, 1924 (1995), and other review articles in this issue

[2] P De Benedetti, Metastable Liquids (Princeton University Press, Princeton, New Jersey, 1997)

[3] C A Angell, J. Phys. Chem. Solids 49, 863 (1988)

[4] H Vogel, Z. Phys. 22, 645 (1921)

G S Fulcher, J. Am. Ceram. Soc. 8, 339 (1925)

[5] W Kauzmann, Chem. Rev. 48, 219 (1948)

[6] G Adams and J H Gibbs, J. Chem. Phys. 43, 139 (1965)

[7] For reviews of mode coupling theories, see W Götze in Liquids, freezing and the glass transition edited by D Levesque, J P Hansen and J Zinn-Justin (Elsevier, New York, 1991)

S P Das, Rev. Mod. Phys. 76, 785 (2004)

[8] For a review of experiments with comparisons to predictions of mode coupling theories, see Dynamics of disordered materials edited by D Richter, A J Dianoux, W Petry and J Teixeira (Springer, Berlin, 1989)

[9] T R Kirkpatrick and P G Wolynes, Phys. Rev. A35, 3072 (1987); Phys. Rev. B36, $8552(1987)$ 
[10] T R Kirkpatrick and D Thirumalai, Phys. Rev. B36, 5388 (1987); Phys. Rev. A37, 4439 (1998); Phys. Rev. B37, 5342 (1988); J. Phys. A22, L149 (1989)

[11] D Thirumalai and T R Kirkpatrick, Phys. Rev. B38, 4881 (1988)

[12] T R Kirkpatrick, D Thirumalai and P G Wolynes, Phys. Rev. A40, 1045 (1989)

[13] R Monasson, Phys. Rev. Lett. 75, 2847 (1995)

[14] G Parisi in Complex behaviour of glassy systems: Proceedings of the XIV Sitges Conference edited by M Rubi and C Perez-Vicente (Springer, Berlin, 1997)

[15] G Parisi, J. Phys. A30, L765 (1997); Phys. Rev. Lett. 79, 3660 (1997)

[16] S Franz and G Parisi, Phys. Rev. Lett. 79, 2486 (1997)

[17] M T Cicerone and M D Ediger, J. Chem. Phys. 103, 5684 (1995)

[18] M Hurley and P Harrowell, Phys. Rev. E52, 1694 (1995)

[19] W Kob, C Donati, S J Plimpton, P H Poole and S C Glotzer, Phys. Rev. Lett. 79, 2827 (1997)

[20] C Dasgupta, A V Indrani, S Ramaswamy and M K Phani, Europhys. Lett. 15, 307 (1991)

[21] C Dasgupta and S Ramaswamy, Physica A186, 314 (1992)

[22] C Donati, S Franz, S C Glotzer and G Parisi, Philos. Mag. B79, 1827 (1999)

[23] J P Bouchaud and M Mézard, J. Phys. I (France) 4, 1109 (1994)

[24] L F Cugliandolo, J Kurchan, G Parisi and F Ritort, Phys. Rev. Lett. 74, 1012 (1995)

[25] C Brangian, W Kob and K Binder, Europhys. Lett. 59, 546 (2002)

[26] X Xia and P G Wolynes, Phys. Rev. Lett. 86, 5526 (2001)

[27] J P Bouchaud and G Biroli, cond-mat/0406317

[28] M Goldstein, J. Chem. Phys. 51, 3728 (1969)

[29] F H Stillinger and T A Weber, Phys. Rev. A25, 978 (1982); Phys. Rev. A28, 2408 (1983)

[30] F H Stillinger, Science 267, 1935 (1995) and references therein

[31] S Sastry, P G Debenedetti and F H Stillinger, Nature 393, 554 (1998)

[32] L Angelani, G Parisi, G Ruocco and G Viliani, Phys. Rev. Lett. 81, 4648 (1998)

[33] F Sciortino, W Kob and P Tartaglia, Phys. Rev. Lett. 83, 3214 (1999)

[34] S Sastry, Phys. Rev. Lett. 85, 590 (2000)

[35] S Buchner and A Heuer, Phys. Rev. Lett. 84, 2168 (2000)

[36] Q Yan, T S Jain and J J de Pablo, Phys. Rev. Lett. 92, 235701 (2004)

[37] See, for example, C De Michele, F Sciortino and A Coniglio, cond-mat/0405282

[38] L Angelani, R D Leonardo, G Ruocco, A Scala and F Sciortino, Phys. Rev. Lett. 85, $5356(2000)$

[39] T S Grigera, A Cavagna, I Giardina and G Parisi, Phys. Rev. Lett. 88, 055502 (2002)

[40] S Sastry, Nature 409, 164 (2001)

[41] W Kob and J-L Barrat, Phys. Rev. Lett. 78, 4581 (1997)

[42] F H Stillinger, J. Chem. Phys. 88, 7818 (1988)

[43] B Doliwa and A Heuer, Phys. Rev. E67, 030501 (2003)

[44] B Doliwa and A Heuer, Phys. Rev. E67, 031506 (2003)

[45] B Doliwa and A Heuer, Phys. Rev. Lett. 91, 235501 (2003)

[46] G H Fredrickson and H C Andersen, Phys. Rev. Lett 53, 1244 (1984)

[47] W Kob and H C Andersen, Phys. Rev. E48, 4364 (1993)

[48] J P Garrahan and D Chandler, Phys. Rev. Lett. 89, 035704 (2002)

[49] L Berthier and J P Garrahan, Phys. Rev. E68, 041201 (2003), and references therein

[50] C Toninelli, G Biroli and D S Fisher, Phys. Rev. Lett. 92, 185504 (2004)

[51] G Biroli, J P Bouchaud and G Tarjus, cond-mat/0412024

D Chandler and J P Garrahan, cond-mat/0501544 
[52] D Kivelson, S A Kivelson, X Zhao, Z Nussinov and G Tarjus, Physica A219, 27 (1995)

[53] L Chayes, V J Emery, S A Kivelson, Z Nussinov and G Tarjus, Physica A225, 129 (1996)

[54] Z Nussinov, J Rudnick, S A Kivelson and L N Chayes, Phys. Rev. Lett. 83, 472 (1999)

[55] Z Nussinov, Phys. Rev. B69, 014208 (2004)

[56] Y Singh, J P Stoessel and P G Wolynes, Phys. Rev. Lett. 54, 1059 (1985)

[57] H Lowen, J. Phys. Condens. Matter 2, 8477 (1990)

[58] C Kaur and S P Das, Phys. Rev. Lett. 86, 2062 (2001)

[59] K Kim and T Munakata, Phys. Rev. E68, 021502 (2003)

[60] T V Ramakrishnan and M Yussouff, Phys. Rev. B19, 2275, (1979)

[61] J P Hansen and I R McDonald, Theory of simple liquids (Academic, London, 1986)

[62] C Dasgupta, Europhys. Lett. 20, 131 (1992)

[63] L M Lust, O T Valls and C Dasgupta, Phys. Rev. E48, 1787, (1993)

[64] L M Lust, O T Valls and C Dasgupta, Phase Transitions 50, 47 (1994)

[65] C Dasgupta and O T Valls, Phys. Rev. E50, 3916 (1994)

[66] O T Valls and C Dasgupta, Transport Theory and Statistical Physics 24, 1199 (1995)

[67] C Dasgupta and O T Valls, Phys. Rev. E53, 2603 (1996)

[68] C Dasgupta and O T Valls, in Complex behavior of glassy systems edited by M Rubí and C Pérez-Vicente (Springer, Berlin, 1997)

[69] C Dasgupta and O T Valls, Phys. Rev. E58, 801 (1998)

[70] C Dasgupta and O T Valls, Phys. Rev. E59, 3123 (1999)

[71] C Dasgupta and O T Valls, J. Phys. Condens. Matter 12, 6553 (2000)

[72] L V Woodcock and C A Angell, Phys. Rev. Lett. 47, 1129, (1981).

[73] L V Woodcock, Ann. N.Y. Acad. Sci. 371, 274, (1981)

[74] P Chaudhuri, S Karmakar and C Dasgupta, manuscript in preparation

[75] M Mézard, G Parisi and M A Virasoro, Spin glass theory and beyond (World Scientific, Singapore, 1987)

[76] G I Menon and C Dasgupta, Phys. Rev. Lett. 73, 1023 (1994)

[77] A Sengupta, S Sengupta and G I Menon, cond-mat/0410390

[78] M Mézard and G Parisi, J. Phys. A29, 6515 (1996)

[79] F Thalmann, C Dasgupta and D Feinberg, Europhys. Lett. 50, 54 (2000)

[80] C Dasgupta and O T Valls, Phys. Rev. E62, 3648 (2000)

[81] M Cardenas, S Franz, G Parisi, J. Phys. A31, L163 (1998); J. Chem. Phys. 110, 1726 (1999)

[82] M Mézard and G Parisi, Phys. Rev. Lett. 82, 747 (1999); J. Chem. Phys. 111, 1076 (1999)

[83] M Mézard, Physica A265, 352 (1999)

[84] B Coluzzi, M Mézard, G Parisi and P Verrocchio, J. Chem. Phys. 111, 9039 (1999)

[85] B Coluzzi, G Parisi and P Verrocchio, J. Chem. Phys. 112, 2933 (2000); Phys. Rev. Lett. 84, 306 (2000)

[86] F Thalmann, J. Chem. Phys. 116, 3378 (2002)

[87] S S Ghosh and C Dasgupta, Phys. Rev. Lett. 77, 1310 (1996)

[88] K Kim and R Yamamoto, Phys. Rev. E61, R41 (2000)

[89] C Brangian, W Kob and K Binder, Europhys. Lett. 53, 756 (2001)

[90] B Doliwa and A Heuer, cond-mat/0210121 